\title{
EEG Feature Variations under Stress Situations
}

\author{
Manuel Merino ${ }^{1}$, Isabel Gómez ${ }^{1}$, Alberto J. Molina ${ }^{1}$
}

\begin{abstract}
The goal of this study is to identify EEG parameters and electrode positions with the highest significant values to differentiate between tasks and relax periods. Different signals were recorded as 12 subjects are doing arithmetic and memory tasks under stress condition. The test consisted of an initial and final 5-minute relax periods and three 4-minute performance phases with increased stress level. $\theta$ and $\alpha$ bands concentrated mainly features whose variation were significant, and F3 and $P 4$ were the best positions to distinguish between performed tasks and arousal level.
\end{abstract}

\section{INTRODUCTION}

Nowadays, interaction with most computing systems does not take into account the state of the users operating them, responding identically to different users or their emotional state. Overcoming this obstacle is the goal of Affective Computing (AC) that has been a promising research field since the end of the last century. AC can be defined as using emotional and contextual information of the user, such as facial expression, nonverbal features of speech, etc., to modify the behavior of an application [1]. A subfield in AC is Physiological Computing (PC) based on data from the human body and how it changes to "provide one means of monitoring, quantifying and representing the context of the user to the system in order to enable proactive and implicit adaptation in real-time" [2].

This intelligent technology can be used in many different fields to improve the adaptive capability of a system or reducing negative feelings like stress [3]-[5], such that determining the subject's emotional state is main task. Some research has concentrated on identifying these states as arousal, stress, workload and/or cognitive-mental load [6]-[9]. They have tried to establish the effect of various psychological states with diverse physiological elements (many-to-many relationship), to determine how several emotional states affect a unique body measure (one-to-many relationship), or gauge the influence of a psychological state on different physiological data (many-to-one relationship) [10]. Thus, when designing an $\mathrm{AC}$ system one has to determine how the task modifies body parameters.

EEG signal are mainly split in frequency five bands: $\delta$ band $(<4 \mathrm{~Hz})$ associates to deep asleep; $\theta$ band $(4-8 \mathrm{~Hz})$ links with the drowsiness and asleep states; $\alpha$ band $(8-14 \mathrm{~Hz})$ shows acitivity with awake, relaxed, no-open-eye states, mainly in occipital area; $\beta$ band $(14-30 \mathrm{~Hz})$ connects to states of activity, mainly frontal and center area of brain; $\gamma$ band $(>30 \mathrm{~Hz})$ is related with information processing. $\theta$ and $\alpha$

\footnotetext{
${ }^{1}$ Electronic Technology Department, University of Seville, Avd. Reina Mercedes s/n, 41012, Seville, Spain. manmermon@dte.us.es; \{igomez, almolina\}@us.es
}

\begin{tabular}{|l|l|l|l|}
$\stackrel{5 \text { minutes }}{ }$ \\
\hline Initial Rest & Phase 1 & Phase 2 & Phinutes \\
\hline
\end{tabular}

Fig. 1. Trial Timing

bands may be used like attention index, workload and level of activity [9], [11]-[13]. Cognitive and workload processes have been associated to large power in the highest segments of the $\alpha$ band $(10-12 \mathrm{~Hz})$, whereas desynchronization of the lowest segments $(8-10 \mathrm{~Hz})$ were assigned to level of attention [14]. Also, increasing memory activity have been related with decreasing the powre in $\theta$ and $\alpha$ bands [15]. $\theta$ power increasing in the middle frontal area was reported during memory and codification periods of new information [16]. $\delta$ and $\beta$ bands decreasing were related with the cognitive aspects of a task [17], and $\gamma$ band increasing was linked with changes of attention, in special in the parietal cortex [18].

This paper attempts to establish a one-to-one relationship focus on EEG signal and stress state, because it has been identified as the second cause of occupational health problems. This work is framed in a study in which we are interested in knowing how EEG features change under stressful situations. This knowledge will allow us to identify the subset of parameters and sensor positions for identifying stress reliable way. In the next section, we describe the experimental protocol (Section 2). Sections 3 and 4 present the experimental results and conclusions.

\section{Methology}

The experimentation took place in a room with artificial lighting and comfortable temperature. Each subject was asked to attend two 22-minute sessions with one week elapsing between them. Subjects were randomly grouped into two subsets. Subset 1 performed the arithmetic task in the first week and the memory task in the second, while subset 2 performed the tasks in reverse order.

Experiments were based on others conducted to analyze stress [6], [8], [19]. Each session was split in 5 parts: the initial and final rest periods of 5 minutes and three 4-minute phases (Fig. 1) in which the task had to be completed. Subjects became accustomed to the task in the first phase, and the level of difficulty and stress were increased in the following phases. Phase 3 should have the greatest level of stress.

Two questionnaires based on the standard State-Trait Anxiety Inventory (STAI) were filled in at the beginning of each relaxation phase [20], where the subject relaxed reading a 


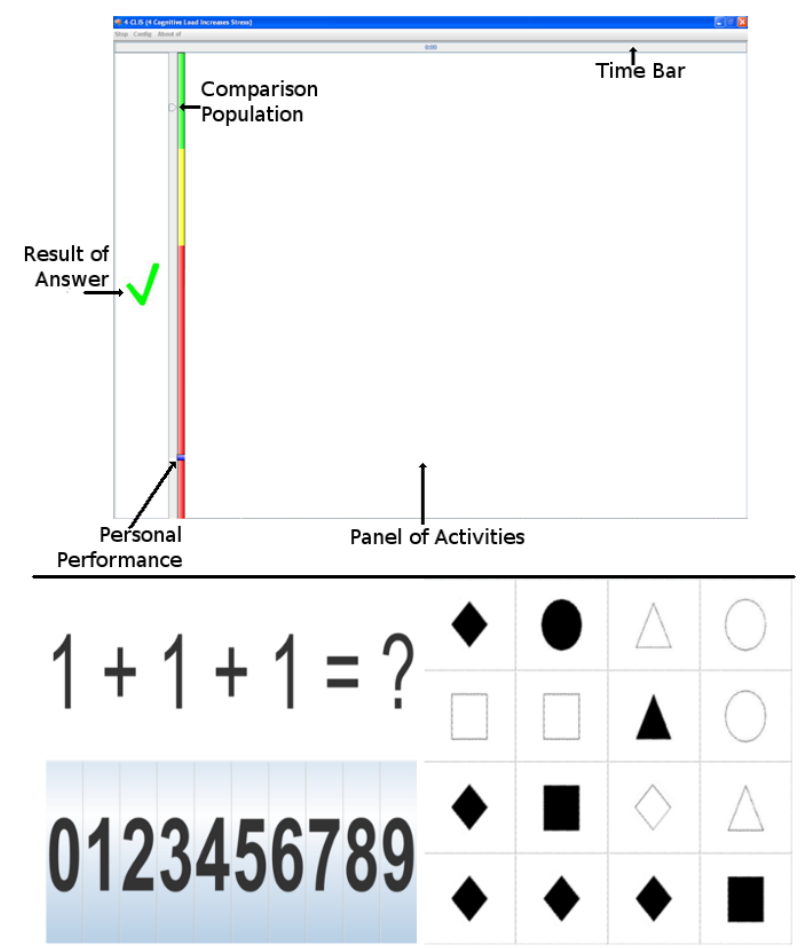

Fig. 2. Trial application. On the top: the Main Application Window showing the panel for memory and arithmetic activities. In the down-left, the arithmetic panel and on the down-right, the memory panel.

magazine. The range of results of testing was between 0 and 60 with the minimum and maximum values indicating total stress/anxiety and complete relaxation.

A Java application was developed to implement the different tasks the subjects had to carry out [19] (Fig. 2). The application screen has four areas: time bar, performance bar with two indicators (comparing user performance with 1.5 times the population result average), answer panel that shows 1 out of 3 messages (correct/non-correct/time out) and a task panel showing the activity. The arithmetic task is based on the Montreal Imaging Stress Task (MIST) [6] where the subjects perform basic math operations (add, subtract, multiplication) whose results are always in the range between $0-9$. The memory activity consists of a matrix where each cell contains a black or white geometric figure (circle, square, triangle, diamond). The individual must memorize the geometric figure, its color and position and then fill in an empty $2 \times 3$ matrix.

In Phase 1, or accommodation period, the subjects grew accustomed to the task. This phase is free of pressure and the performance bar is not shown. The average of the results for the population was calculated with the correct answers for this period (the subjects were not aware of this) to determine the population comparison indicator.

In Phase 2, each subject was asked to try and exceed the population result indicator, being told that otherwise the data could not be used to compute the average for other individuals. The mental stress and arousal were therefore higher in this phase.

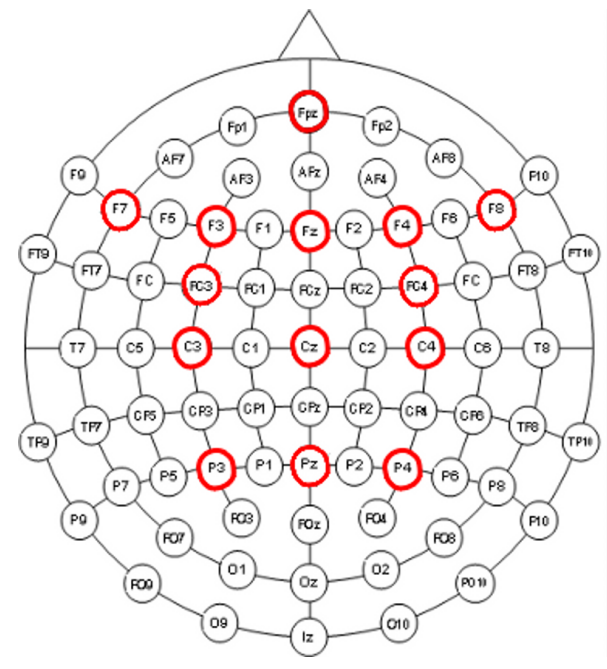

Fig. 3. EEG electrode scheme.

In Phase 3, the researcher introduced stressors by telling the subject that the data from the last phase was useless because he/she had not achieved the goal, unlike the rest of the subjects who had passed the phase correctly. Even more stress was added by asking questions such as"Did you sleep well?", "Do you have personal problems?", etc. and making comments such as "You've got it wrong", "You must concentrate", "Time's running out".

\section{A. Subjects}

The trials were conducted with 13 healthy subjects aged between 26-56 (mean 37.86; sd 9.93), two of them were women and eleven men. All of them were voluntary and they work in our same place. Twelve subjects completed the arithmetic task, while eleven performed the memory task. Ethics committee of the University of Seville approved this research.

\section{B. Data Acquisition}

The biosignal amplifier was set at $256 \mathrm{~Hz}$, with a Chebyshev notch filter $(48,52) \mathrm{Hz}$ of order 4 to delete electrical power signals. A bandpass filter of $(4,45) \mathrm{Hz}$ and a correlation filter with EOG data as reference signal were applied to remove environment noise, drifts, and EOG interference. Visual inspection was done to delete segments with significant head or body movements interference.

Selected EEG standard positions were: Fpz, F7, F3, Fz, F4, F8, FC3, FC4, C3, Cz, C4, P3, and P4 (Fig. 3), where $\mathrm{Fpz}$ was utilized as ground and $\mathrm{Cz}$ was used as reference sensor.

The offline data analysis was done with version 7.6.0.324 of Matlab.

\section{EEG Parameters}

EEG signal was divided in four frequency bands: (4, $8] \mathrm{Hz}$ is $\theta,(8,14] \mathrm{Hz}$ is $\alpha,(14,30] \mathrm{Hz}$ is $\beta$, and $>30 \mathrm{~Hz}$ is $\gamma$. Thrity two parameters were calculated: band energy $\left(E_{\theta}\right.$, $\left.E_{\alpha}, E_{\beta}, E_{\gamma}\right)$, standard deviation of each band $\left(s t d_{\theta}\right.$, st $d_{\alpha}$, 

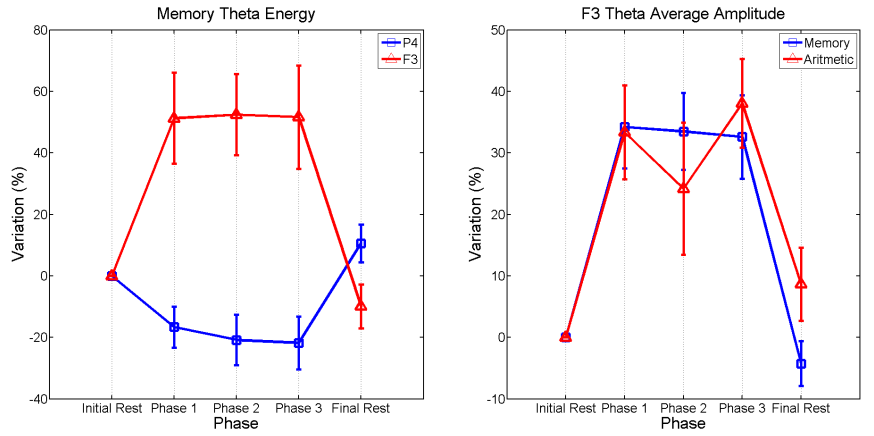

Fig. 4. Feature variations with initial rest as refline. On the left is showed $\theta$ energy in F3 and P4 for memory task. On the right is drew $\theta$ amplitude mean in F3 for both activities.

$\left.s t d_{\beta}, s t d_{\gamma}\right)$, spectral amplitude average $\left(\overline{A S_{\theta}}, \overline{A S_{\alpha}}, \overline{A S_{\beta}}\right.$, $\left.\overline{A S_{\gamma}}\right)$, spectral centroid $\left(f_{\theta}, f_{\alpha}, f_{\beta}, f_{\gamma}\right)$, percentage of each bands in total energy $\left(I_{\theta}, I_{\alpha}, I_{\beta}, I_{\gamma}\right)$, the logarithm of ratio between $E_{\beta}$ and the sum of $E_{\theta}$ and $E_{\alpha}\left(E E G_{\omega}\right.$ [5]), and from total EEG signal, in time domain, total energy $\left(E_{E E G}\right)$, fractal dimension $(F D)$ using Higuchi method [9], six order AR model coefficients $\left(A R_{j}, j \in \mathbb{Z}=\{1,6\}\right)$, and Hjorth's parameters [21], that is, activity $(A c)$, movility $(M)$ and complexity $(C O M)$.

\section{RESULT}

We are not just interested in discovering how the different features change through the phases for a specific subject, but also in choosing the best features for detecting the onset of a stressful situation for a population as a whole. After obtaining the features for the set of subjects in each phase we studied the Wilcoxon signed rank test with 0.05 statistical significance for each phase respect using initial rest as refline and for activity phases versus accommodation period (phase 1). ANOVA analysis was rejected because some features did not fulfill the normal-distribution requirements (Shapiro test).

On the other hand, a variation analysis between phases were obtained by applying the equation (1), where $\Delta J_{r e f}^{i}$ is the variation of the parameter $J$ in the phase $i$ with phase ref as refline; $\Delta J^{i}$ and $\Delta J^{\text {ref }}$ are the values of the parameter $J$ in the phase $i$ and $r e f$ where $i \neq r e f$.

$$
\Delta J_{r e f}^{i}=100 \cdot\left(\left(J^{i} / J^{r e f}\right)-1\right) i \neq r e f
$$

The expected behavior for the change from the initialrest period to the first task phase is a change resulting from performance period, since the subject passes from an initial relaxed state to a mental-activity period and because the individual does not know how the task and the control of the application work. So, a significant difference between task phases and rest periods is the a priori expected behavior (PEB), making it possible to distinguish between the activity and relaxed states. Rest periods have to show non-significant differences. This is the goal of the first statistic analysis. The parameters which do not show those changes must be rejected as correct indicators of stress level, even if one of the phases has a significant change. On the other hand, stress
TABLE I

FIRST STATISTIC ANALYSIS RESULTS ( $a=$ PEB IN ARITHMETIC TASK, $m=$ PEB IN MEMORY TASK, $\cap=$ VARIATION IN INVERTED-U SHAPED, $U=$ VARIATION IN U SHAPED).

\begin{tabular}{|l|l|l|l|l|l|l|l|l|l|l|l|l|}
\hline Feature & F7 & F3 & Fz & F4 & F8 & FC3 & FC4 & C3 & C4 & P3 & P4 & Total \\
\hline
\end{tabular}

\begin{tabular}{|c|c|c|c|c|c|c|c|c|c|c|c|}
\hline$A c$ & & & & & & & & & & $\mathrm{~m} \cup$ & $1 m$ \\
\hline COM & $\mathrm{m} \cap$ & & & & & & $\mathrm{a} \cup$ & & & & $\begin{array}{l}1 a \\
1 m\end{array}$ \\
\hline$E E G_{\omega}$ & $\mathrm{m} \cap$ & & & & & & & & & & $1 m$ \\
\hline$f_{\theta}$ & $\mathrm{mU}$ & $\mathrm{m} \cup$ & $\mathrm{m} \cup$ & & & & & & $\mathrm{mU}$ & $\mathrm{mU}$ & $5 m$ \\
\hline$f_{\alpha}$ & & & & & & & & & $a \cap$ & $\mathrm{a} \cap$ & $2 a$ \\
\hline$I_{\Theta}$ & $\mathrm{m} \cap$ & $\mathrm{m} \cap$ & & & & & & & & & $2 m$ \\
\hline$I_{\alpha}$ & & & & & & & & & $\mathrm{m} \cup$ & & $1 \mathrm{~m}$ \\
\hline$I_{\beta}$ & $\mathrm{mU}$ & $\mathrm{mU}$ & & & & & & & & & $2 m$ \\
\hline$s t d_{\theta}$ & $\mathrm{m} \cap$ & $\mathrm{m} \cap$ & $\mathrm{m} \cap$ & & $\mathrm{m} \cap$ & & & & & & $4 m$ \\
\hline$s t d_{\alpha}$ & $\begin{array}{l}\mathrm{a} \cap \\
\mathrm{m} \cap\end{array}$ & $a \cap$ & & & & & & & & & $\begin{array}{l}2 a \\
1 m\end{array}$ \\
\hline$s t d_{\beta}$ & & & & & & & $a \cap$ & & & & $1 a$ \\
\hline$s t d_{\gamma}$ & & & & & & & $a \cap$ & & & & la \\
\hline$E_{\theta}$ & & & $\mathrm{m} \cap$ & & & & & & & $\mathrm{m} \cup$ & $2 m$ \\
\hline$E_{\beta}$ & & & & & & & & & & $\mathrm{m} \cup$ & $1 m$ \\
\hline$\frac{p}{E_{E E G}}$ & & & & & $\mathrm{~m} \cup$ & $\mathrm{m} \cup$ & & $\mathrm{m} \cup$ & & $\mathrm{m} \cup$ & $4 m$ \\
\hline$\overline{A S_{\Theta}}$ & $\begin{array}{l}\mathrm{a} \cap \\
\mathrm{m} \cap\end{array}$ & $\begin{array}{l}\mathrm{a} \cap \\
\mathrm{m} \cap\end{array}$ & $\begin{array}{l}\mathrm{a} \cap \\
\mathrm{m} \cap\end{array}$ & $a \cap$ & $\mathrm{m} \cap$ & $a \cap$ & & & & & $\begin{array}{l}5 a \\
4 m\end{array}$ \\
\hline$\overline{A S_{\alpha}}$ & $\begin{array}{l}\mathrm{a} \cap \\
\mathrm{m} \cap\end{array}$ & $\mathrm{a} \cap$ & & & & & & & & & $\begin{array}{l}2 a \\
1 m\end{array}$ \\
\hline$\overline{A S_{\beta}}$ & & $a \cap$ & & & & & & & & & $1 a$ \\
\hline$\overline{A S_{\gamma}}$ & & & & & & & $a \cap$ & & & & $1 a$ \\
\hline Total & $\begin{array}{l}3 a \\
9 m\end{array}$ & $\begin{array}{l}4 a \\
5 m\end{array}$ & $\begin{array}{l}1 a \\
4 m\end{array}$ & $1 a$ & $3 m$ & $\begin{array}{l}1 \mathrm{la} \\
1 \mathrm{~m}\end{array}$ & $4 a$ & $1 m$ & $\begin{array}{c}1 a \\
2 m\end{array}$ & $\begin{array}{l}19 \\
5 m\end{array}$ & \\
\hline
\end{tabular}

level should be confirmed from the second statistic analysis, so that, the stress level is confirmed these variations occur in second and third performance phases compared to first activity phase.

\section{A. STAI Test}

As we mentioned earlier, the subjects were given two stress tests during the initial and final rest phases for arithmetic and memory tasks. The difference between the final and initial STAI scores gave us information about whether or not the task was stressful. Negative differences indicated that the task was stressful, whatever the experimental situation for both tasks. Specifically, in the arithmetic task, the average difference was -7.5 with a standard error of $2.18 \%$, and in the memory task this difference was -5.0 with a standard error of $1.46 \%$.

\section{B. Electroencephalogram}

Table I summarizes first statistic analysis results where PEB was found. EEG positions with more PEB features were $\mathrm{F} 3, \mathrm{Fz}$ and $\mathrm{C} 3$ for arithmetic activity, and F3, Fz, and P4 in memory performance. F8, C3 in memory, FC3, C4 in arithmetic, and $\mathrm{F} 7$ for both tasks have not shown any PEB features. All arithmetic features showed variations in inverted-U shaped, except COM, while in memory, F3 and P4 showed inverse variations, such that majority of them drew a shape like inverted- $U$ and $U$ respectively. An inverted-U and $\mathrm{U}$ shapes mean how are feature changes, so that increasing and decreasing of parameter during performance is inverted$\mathrm{U}$ and $\mathrm{U}$ respectively. An example is showed in Fig. 4. 
Second statistic analysis showed non-significant changes of all features respect to first activity phase, whereas, in memory, significant variations were obtained in $A c$ in $\mathrm{P} 4$ during second performance, and $\overline{A S_{\theta}}$ in FC3 and $\overline{A S_{\alpha}}$ in F3 during third activity phase. Thus, only these parameters could be used as stress index. However, stress-4-minute period may not be enough to achieve stressed EEG data, against used biosignals in others studies, like electrodermal activity or electrocardiogram [8], [19].

$\theta$ and $\alpha$ bands causes mainly PEB features in $\mathrm{F}$ positions, mainly left area (logical, arithmetic area), such that their values increase with activities what can be observed with spectral amplitude, its dispersion, and influence and frequency centroid of $\theta$ [9], [11]-[16]. Cognitive task aspects show inversed behavior of $I_{\theta}$ and $I_{\beta}$, and $\overline{A S_{\theta}}$ increasing are linked with previous results from [16], [17]. $\theta$ frequency centroid shifts towards $\delta$ band with activity. This fact may be related with $\delta$ power increasing reported in [17]. On the other hand, reports of spectral dispersion were not found.

\section{CONClusion And Future Work}

This study is focused on selecting EEG positions and parameters. Activity effects are observed in EEG features, three of them showed stress changes and it is confirm with a questionnaire. However, stressful period should be longer to get more features. $\theta$ and $\alpha$ bands congregate the majority of PEB parameters. F3, Fz and F4 electrode positions concentrate mainly these variations, while F7, F8, FC3 and C4 could be rejected as good activity/stress locations. Also, F3 is a common position in both tasks, and P4 is mainly linked with memory performance. Next step is to develop a stress and task classifier based on these results.

\section{REFERENCES}

[1] R. El Kaliouby, R. Picard, and S. Baron-Cohen, "Affective computing and autism," Affiliation: Massachusetts Institute of Technology, Cambridge, MA 02142-1308, United States; Affiliation: University of Cambridge, Cambridge CB3 0FD, United Kingdom; Affiliation: FIEEE, MIT Media Laboratory, E15-448, 20 Ames Street, Cambridge, MA 02142-13, pp. 228-248, 2006.

[2] S. H. Fairclough, "Fundamentals of physiological computing," Interacting with Computers, vol. 21, no. 1-2, pp. 133-145, 2009.

[3] F. Nasoz, C. L. Lisetti, and A. V. Vasilakos, "Affectively intelligent and adaptive car interfaces," Information Sciences, vol. 180, no. 20, pp. 3817-3836, 2010.

[4] R. R. Singh, S. Conjeti, and R. Banerjee, "An approach for realtime stress-trend detection using physiological signals in wearable computing systems for automotive drivers," in 14th IEEE International Intelligent Transportation Systems Conference, ITSC 2011. Affiliation: Department of Electrical and Electronics Engineering, Birla Institute of Technology and Science (BITS) Pilani, Rajasthan, 333031, India; Affiliation: Department of Computer Science, Birla Institute of Technology and Science (BITS) Pilani, Raj, 2011, pp. 1477-1482.

[5] G. Chanel, C. Rebetez, M. Bétrancourt, and T. Pun, "Emotion assessment from physiological signals for adaptation of game difficulty," IEEE Transactions on Systems, Man, and Cybernetics Part A:Systems and Humans, vol. 41, no. 6, pp. 1052-1063, 2011.

[6] K. Dedovic, R. Renwick, N. K. Mahani, V. Engert, S. J. Lupien, and J. C. Pruessner, "The Montreal Imaging Stress Task: Using functional imaging to investigate the effects of perceiving and processing psychosocial stress in the human brain," Journal of Psychiatry and Neuroscience, vol. 30, no. 5, pp. 319-325, 2005.

[7] V. V. Lazarev, "The relationship of theory and methodology in EEG studies of mental activity," International Journal of Psychophysiology, vol. 62, no. 3, pp. 384-393, 2006.
[8] C. Setz, B. Arnrich, J. Schumm, R. L. Marca, G. Tr, and U. Ehlert, "Using a Wearable EDA Device," vol. 14, no. 2, pp. 410-417, 2010.

[9] Q. Wang and O. Sourina, "Real-time mental arithmetic task recognition from EEG signals," IEEE Transactions on Neural Systems and Rehabilitation Engineering, vol. 21, no. 2, pp. 225-232, 2013.

[10] J. T. Cacioppo and L. G. Tassinary, "Inferring psychological significance from physiological signals." The American psychologist, vol. 45, no. 1, pp. 16-28, 1990.

[11] Y. Maki, G. Sano, Y. Kobashi, T. Nakamura, M. Kanoh, and K. Yamada, "Estimating subjective assessments using a simple biosignal sensor," Proceedings - 13th ACIS International Conference on Software Engineering, Artificial Intelligence, Networking, and Parallel/Distributed Computing, SNPD 2012, pp. 325-330, 2012.

[12] M. Azarnoosh, A. Motie Nasrabadi, M. R. Mohammadi, and M. Firoozabadi, "Investigation of mental fatigue through EEG signal processing based on nonlinear analysis: Symbolic dynamics," Chaos, Solitons \& Fractals, vol. 44, no. 12, pp. 1054-1062, 2011.

[13] E. Wascher, B. Rasch, J. Sänger, S. Hoffmann, D. Schneider, G. Rinkenauer, H. Heuer, and I. Gutberlet, "Frontal theta activity reflects distinct aspects of mental fatigue," Biological Psychology, vol. 96, no. 1, pp. 57-65, 2014.

[14] W. Klimesch, "EEG alpha and theta oscillations reflect cognitive and memory performance: a review and analysis." Brain research. Brain research reviews, vol. 29, no. 2-3, pp. 169-195, 1999.

[15] A. Gevins, M. E. Smith, L. McEvoy, and D. Yu, "High-resolution EEG mapping of cortical activation related to working memory: Effects of task difficulty, type of processing, and practice," Cerebral Cortex, vol. 7, no. 4, pp. 374-385, 1997.

[16] A. Gevins, M. E. Smith, H. Leong, L. McEvoy, S. Whitfield, R. Du, and G. Rush, "Monitoring working memory load during computerbased tasks with EEG pattern recognition methods." Human factors, vol. 40, no. 1, pp. 79-91, 1998.

[17] T. Fernández, T. Harmony, M. Rodríguez, J. Bernal, J. Silva, A. Reyes, and E. Marosi, "EEG activation patterns during the performance of tasks involving different components of mental calculation." Electroencephalography and clinical neurophysiology, vol. 94, no. 3, pp. $175-182,1995$.

[18] T. Gruber, M. M. Müller, A. Keil, and T. Elbert, "Selective visualspatial attention alters induced gamma band responses in the human EEG," Clinical Neurophysiology, vol. 110, no. 12, pp. 2074-2085, 1999.

[19] M. M. Monge, I. Gomez, and A. Molina, "Stress and heart rate : significant parameters and their variations Article Type : original article," Exp Clin Cardiol, vol. 20, no. 8, pp. 3509-3517, 2014.

[20] C. D. Spielberger, R. L. Gorsuch, P. R. Lushene, P. R. Vagg, and A. G. Jacobs, Manual for the State-Trait Anxiety Inventory (Form Y), 1983.

[21] B. Hjorth, "EEG analysis based on time domain properties," pp. 306$310,1970$. 